\title{
The FIP Effect and Abundance Anomalies in Late-Type Stellar Coronae
}

\author{
J. J. DRAKE, ${ }^{1}$ J. M. LAMING, ${ }^{2}$ AND K. G. WIDING ${ }^{3}$ \\ ${ }^{1}$ Center for EUV Astrophysics, 2150 Kittredge Street, \\ University of California, Berkeley, CA 94720-5030, USA \\ ${ }^{2}$ SFA Inc., Landover, MD 20785, and Naval Research Laboratory, \\ Code $7674 \mathrm{~L}$, Washington, DC 20375, USA \\ ${ }^{3} \mathrm{Naval}$ Research Laboratory, Code $7674 \mathrm{~W}$, Washington, DC 20375, USA
}

\begin{abstract}
"Yes, it will be a long time before people know what I know. How much of iron and other metal there is in the sun and the stars is easy to find out, but anything which exposes our swinishness is difficult, terribly difficult" (Tolstoy 1889).

In the solar corona, the abundances of elements appear to differ from the photospheric values in a manner related to the element first ionization potential (FIP): species with FIP $\leq 10 \mathrm{eV}$ are observed to be enhanced relative to the photosphere by factors of $\sim 3-4$. The first studies of stellar coronal composition with EUVE suggest that some stars exhibit a solar-like FIP effect, whereas others do not. We briefly review the latest results, and we argue that element abundance anomalies, such as the FIP effect, can provide potentially powerful new coronal diagnostics. Moreover, knowledge of the composition of a stellar corona is crucial for interpreting its spectrum-for understanding its structure and energy balance, and for testing its possible heating mechanisms: We must begin to understand coronal abundance anomalies and the compositions of active stars in order to begin to understand their coronal physics.
\end{abstract}

\section{Introduction}

As hinted by Tolstoy (1889), swinish suggestions that the abundances of elements in the solar corona might differ significantly from the corresponding values in the underlying photosphere are not entirely recent. The first hint in modern times came from the pioneering work of Pottasch (1963), who obtained significantly different abundances for some elements, most notably $\mathrm{Mg}, \mathrm{Si}$ and $\mathrm{Fe}$, than the generally accepted photospheric abundances of the day (those of Goldberg, Müller \& Aller 1960). Regarding Mg, Si and $\mathrm{Fe}$, Pottasch stated that the abundance differences could not be explained by experimental uncertainties-conclusions which are not compromised by today's revised solar photospheric abundances. Perhaps more interestingly, Pottasch also remarked that the agreement between his abundances and those observed in cosmic rays was much closer than with the photospheric values.

The story of the study of abundances in the solar corona and wind during the intervening 30 years or so has been comprehensively reviewed by Meyer $(1985 a, b ; 1993)$ and Feldman (1992), $\dagger$ and will also be reviewed briefly elsewhere in this volume (Haisch, Saba \& Meyer 1995; see also Saba 1995 for a review of the Solar Maximum Mission results). In summary, the last three decades have seen the early hints of abundance anomalies raised by the Pottasch analysis grow into a substantial body of evidence that points to a significant abundance anomaly in the solar corona. This anomaly appears to have a common underlying trend related to the element first ionization potential (FIP), similar to that

† Note that the coronal abundances adopted by Feldman (1992) differ from those of Meyer (1985) in the normalization with respect to H: Meyer (1985) suggested that the high FIP species are depleted with respect to $\mathrm{H}$, whereas Feldman argues that the low FIP species are enhanced relative to H; Meyer (1993) tends to side with Feldman's (1992) assessment. 
uncovered for the low to medium energy cosmic rays by Cassé \& Goret (1978): elements with a low FIP ( $\$ 10 \mathrm{eV}-$ e.g., $\mathrm{Mg}, \mathrm{Si}, \mathrm{Fe}$ ) appear to be enhanced relative to elements with high FIP ( $(10 \mathrm{eV}-$ e.g., $\mathrm{O}, \mathrm{Ne}, \mathrm{S}$ ) by average factors of 3-4. The phenomenon is now known as the "FIP Effect," but the sites and mechanisms responsible for this elemental fractionation according to FIP are currently not known or understood. The fractionation energy of $10 \mathrm{eV}$ points to chromospheric temperatures, but a photospheric fractionation site has not been ruled out.

In this short paper, we point out the importance of coronal abundances and review recent results. Studies of abundances in stellar coronae might be extremely valuable for providing key insights into the FIP Effect which solar observations alone cannot. Three important questions of wide astrophysical importance are raised: (1) Do other stars exhibit coronal abundance anomalies? (2) If so, are the anomalies related to FIP, as in the solar case? (3) Is the solar FIP Effect connected to that observed in cosmic rays?

\section{The Importance of Stellar Coronal Abundances}

The flux, $F_{j i}$, emitted through a spectral line $j \rightarrow i$ by an optically thin stellar corona is essentially proportional to the abundance of the element in question. The expression for the observed line flux can be closely approximated by the integral over the temperature interval, $\Delta T_{j i}$, over which the line flux is non-negligible (usually, $\Delta \log T_{j i} \sim 0.3$ ),

$$
F_{j i}=A K_{j i} \int_{\Delta T_{j i}} G_{j i}(T) \overline{n_{e}^{2}}(T) \frac{d V(T)}{d T} d T \mathrm{erg} \mathrm{cm}^{-2} \mathrm{~s}^{-1}
$$

where $A$ is the abundance of the element in question, $K_{j i}$ is a known constant which includes the frequency of the transition and the stellar distance, $n_{e}(T)$ is the number density of electrons at temperature $T$ within the plasma volume $V(T)$, and $G_{j i}(T)$ is the "contribution" function of the line. This last parameter defines the temperature interval, $\Delta T_{j i}$, over which the line is formed, and is dependent on the atomic physics of the particular transition. For most transitions of interest, $G_{j i}(T)$ can be fairly reliably estimated theoretically. The integrand $\overline{n_{e}^{2}}(T) \frac{d V(T)}{d T}$ is the "differential emission measure".

The abundance pattern in the emitting plasma, then, influences both the spectral shape and intensity: deriving basic quantities such as the emission measure cannot be done without prior knowledge of the composition. In short, the plasma composition is fundamental to the basic interpretation and analysis of spectroscopic and photometric observations of stellar outer atmospheres.

Since stellar coronae cannot be spatially resolved, the global emission measure distribution as a function of temperature, hopefully with some clues as to the density for at least one temperature, will form the extent of the tangible data from which we must begin to understand stellar coronal structure. Moreover, radiative loss, together with the emission measure form the key ingredients for estimating the outer atmosphere energy budget, which is a necessary step towards pinpointing and testing possible heating mechanisms. Similarly, the energetics of transient phenomena such as flares and coronal mass ejections cannot be deduced without knowledge of the plasma element abundances, since line emission from species such as $\mathrm{Fe}, \mathrm{Mg}, \mathrm{Si}$ and $\mathrm{O}$ control radiative cooling times and the onset of thermal instabilities (see, e.g., Cook et al. 1989).

A situation in which the abundances change from one coronal structure to another, and vary with time, such as appears to be the case for the solar corona, and in which the underlying patterns of abundance variations might differ widely from one spectral type and activity level to the next, such as is suggested by recent $E U V E$ and $A S C A$ analyses, will pose somewhat of a challenge to those hoping to understand the observations. 
It should also be pointed out that the photospheric compositions are either unknown or extremely uncertain for nearly all of the stars whose coronae are bright enough for detailed spectroscopic study. In particular, the RS CVn's, the algols and the late K and $\mathrm{M}$ dwarf single and binary stars generally do not have reliable abundances and often lack even rough metallicity estimates-measurements are very difficult because of rapid rotation, binarity, and, in the case of the late $K$ and $M$ dwarfs, severe line blanketing. We can probably not expect to know the photospheric compositions of these types of stars to much better than a factor of 2 . Thus, even in the absence of any coronal abundance anomalies, uncertainties in composition still pose significant difficulties for coronal physics.

\subsection{Clues to Coronal Structure and Underlying Physical Processes?}

While compositional uncertainties complicate the interpretation of coronal observations, we speculate that, if the abundance anomalies can be understood, then coronal abundances might provide powerful diagnostics of the physics and structure of stellar coronae. This is prompted by observations of the solar corona which suggest that there are systematic variations of abundances in different types of coronal structure. These observations have been discussed by Feldman (1992) and by Meyer (1993). We can summarize briefly the main findings as follows:

(a) Above sunspots, coronal loops and material have been observed to have photospheric compositions.

(b) In active regions, newly emerged low-lying bipolar loops have photospheric compositions.

(c) In active regions, evolved loops more than 1 day old have enhancements of low FIP species by factors of $\sim 4-5$; no enhancements larger than these factors are observed in regions of closed magnetic fields.

(d) Features associated with closed magnetic fields and compact loop structures, such as young active regions and flares, show photospheric abundance ratios.

(e) Features associated with open unipolar magnetic fields such as polar plumes show extreme coronal type composition. On the other hand the diffuse emission over polar coronal holes, and the fast solar wind, appear to have composition between photospheric and coronal.

To these we add:

(f) The supergranulation network emission has a photospheric composition; the FIP effect appears also to be a function of altitude.

\subsection{Do Cosmic Rays Originate from Late-Type Stellar Coronae?}

The problem of the origin of cosmic rays remains an outstanding question in high energy astrophysics. A possible clue to the galactic cosmic ray sources (GCRS; $\$ 100 \mathrm{GeV}$ ) was uncovered a decade or so ago by Cassé and Goret (1978), whose analysis of their elemental composition revealed the underlying correlation of heavy element abundances with FIP. Subsequently, Meyer (1985b) pointed out that this composition was very similar to that of solar energetic particles and suggested that most GCRs could originate in unevolved late-type star surface material (with some small fraction coming from massive WC stars).

Direct in situ acceleration of stellar coronal or wind material meets with difficulties on energetic grounds (Mullan 1979; Gorenstein 1981). A two-step process was then suggested, whereby some fraction of the $\sim \mathrm{MeV}$ particles "injected" by stellar coronae are boosted to relativistic energies by stochastic or diffusive shock acceleration provided mainly by supernova remnants (e.g., Axford et al. 1977). This model has been successful in producing quite naturally a CR energy spectrum which is consistent with the $C R$ 
source spectrum inferred from observations (e.g. van Bloemen 1987). Moreover, it is particularly appealing since other non-stellar injection models suffer the disadvantage of both having to explain the fractionation through other means, and facing the prospect that the remarkable similarity between the $\mathrm{CR}$ source composition and the composition of solar energetic particles is purely accidental (van Bloemen 1987). Stellar population considerations then suggest that the $K$ and $M$ dwarfs would be the major injectors of suprathermal CR seed particles (Gorenstein 1981). However, this hypothesis is based entirely on extrapolation of the solar coronal abundance case, since, before the launch of $E U V E$, it was not possible to determine the abundances of elements in the coronae of other stars.

\section{Determining Abundances in Stellar Coronae with EUVE}

There are three ways in which $E U V E$ spectra might be used for gleaning compositional information in coronal plasmas, all of which have been previously applied to the Sun.

(a) Comparing the EM predicted by a line of one element with a baseline EM distribution (essentially the "differential emission measure" $n_{e}^{2}(T) \frac{d V(T)}{d T} d T$ of Eqn. 2.1) derived using lines of another element (in practice, Fe). The ratios of the EM's predicted by elements $\mathrm{X}$ and $\mathrm{Y}$ then yield their abundance ratios relative to the composition assumed for the plasma in the EM calculations: for an assumed solar composition, $\log \left(E M_{X} / E M_{Y}\right)$ yields the abundance ratio $[\mathrm{X} / \mathrm{Y}]$, using the conventional spectroscopic bracket notation.

(b) Temperature insensitive line ratios-comparing the fluxes of lines which have approximately the same temperature dependence in their $G_{i j}(T)$ functions: in the expression describing the ratio of the two line fluxes, from Eqn. 2.1, all terms cancel except for the element abundance and the relevant atomic physics parameters. This method does not then require detailed knowledge of the EM distribution.

(c) Line-to-continuum ratios. If the EM distribution is known, the abundance of an element relative to $\mathrm{H}$ can be derived by measuring the strength of an emission line relative to the thermal bremsstralung continuum, which is formed primarily by electron free-free interactions with protons. In order for such measurements to be possible, the continuum needs to be firmly detected. In the case of EUVE observations, this is only likely for the very hot $\left(T \sim 10^{7} \mathrm{~K}\right)$ coronae found in rapidly rotating stars, or for long observations of less active stars (however, see $\S 4.3$ ).

\section{Recent Stellar Coronal Abundance Studies in the EUV}

\subsection{The FIP Effect in the Full Solar Disk}

We have recently investigated the FIP effect in the full disk ("Sun as a star") solar corona (Laming, Drake \& Widing 1995; Figure 1a). At $\log T \sim 6.3$, the low FIP elements are enhanced by about a factor of 3 . However, this enhancement varies with temperature, and at temperatures cooler than $\log T \sim 5.8$-the temperatures dominated by supergranulation emission - there is no discernible FIP effect in the full disk average corona.

Laming, Drake \& Widing (1995) suggested that in full disk spectra, the dominant emission at temperatures of a few $10^{5} \mathrm{~K}$ comes from low lying structures associated with the supergranulation network with essentially no FIP effect, while true coronal emissions comes from active loops and much higher altitudes where the FIP effect is strong. Such an idea of the "discontinuity" between the corona and the transition region has also been discussed by Feldman \& Laming (1994) on the basis of observations of the different 


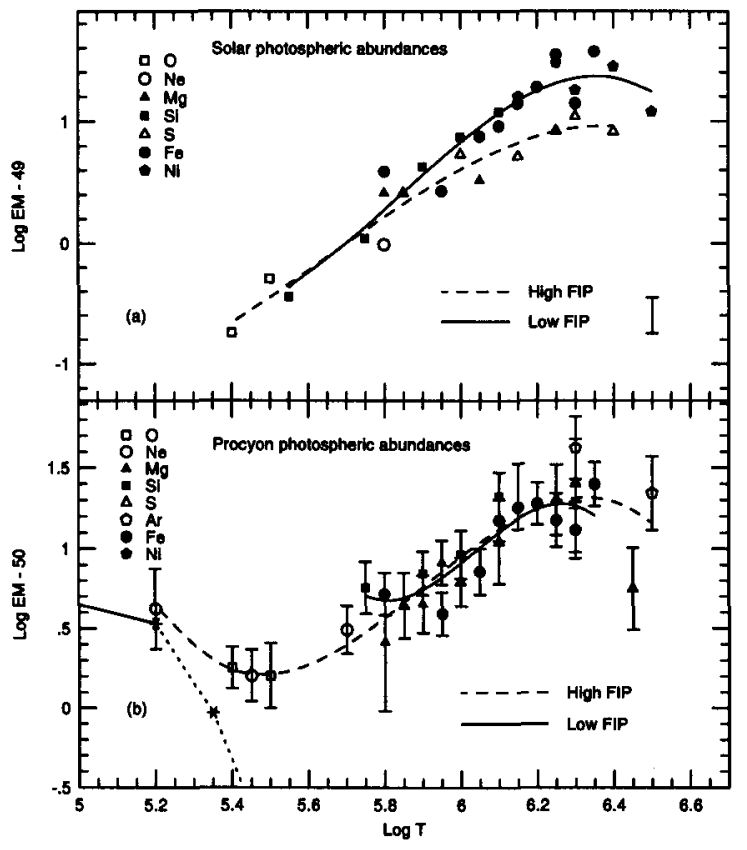

FIGURE 1. EM distributions derived for low and high FIP elements in (a) the solar full disk corona, and (b) the corona of Procyon. The curves illustrate best-fit splines to the high and low FIP points. There appears to be no FIP effect in Procyon, while in the full disk solar corona there is no significant FIP effect for $\log T<5.8$.

in morphology between lines formed at temperatures above and below $\sim 10^{6} \mathrm{~K}$, and the apparent absence of footpoints emitting transition region radiation at the base of otherwise coronal loops.

\subsection{Evidence for No FIP Effect in Procyon-Acoustic Heating or a Photospheric Fractionation Site?}

In a recent analysis of $E U V E$ spectra we have found evidence for photospheric abundances in the corona of the F5 subgiant Procyon (Drake et al. 1995; Drake, Laming \& Widing 1995a; Figure 1b). This result might be an indication that Procyon's corona is mainly heated by acoustic means, as has been suggested by some workers (e.g. Mullan \& Cheng 1994; Simon \& Drake 1989). For diffusion models of element fractionation to work, small spatial scales are required, otherwise, neutral species have sufficient time to become ionized during the separation process (von Steiger \& Geiss 1989). Drake et al. (1995a) pointed out that such small spatial scales can occur quite naturally with magnetic fields, but not with acoustic waves.

Alternatively, the only high FIP lines available in the Procyon spectrum at coronal temperatures are due various charge states of $S$, and two rather unsatisfactory blended lines from Ar XII (224.23) and Ar XV (221.20). If the FIP fractionation site is photospheric, then it is possible that $\mathrm{S}$ (IP $\sim 10 \mathrm{eV}$ ) could be acting as a low FIP species in the hotter $(6500 \mathrm{~K})$ photosphere of Procyon. This hypothesis can be also tested by $E U V E$ observations of stars cooler than the Sun-see below. 


\subsection{Depleted Fe in the Coronal Emission from Algol and CF Tuc?}

Based on line-to-continuum ratios of Fe lines observed in the quiescent Algol EUVE spectrum, Stern et al. (1994) have demonstrated evidence that Fe must be underabundant (relative to the solar photospheric abundances of Anders and Grevesse 1989) by a factor of $\sim 4$. For an object as young as Algol, such an Fe deficiency is not likely; the only obvious alternative is that the corona is deficient in $\mathrm{Fe}$. The case for a low coronal $\mathrm{Fe}$ abundance was first suggested by the $A S C A$ observations of Antunes et al. (1994), who derived an abundance for $\mathrm{Fe}$ of $32 \%$ of the solar value (see also $\S 4.5$ ).

More extreme than the Algol case is that of the RS CVn system CF Tuc, whose EUVE spectrum exhibits almost no lines! Schmitt et al. (1995) have shown that most of the EUV flux from CF Tuc is in the form of continuum. No plausible EM distribution can explain the absence of the strong Fe lines in charge states XVIII-XXIV expected from such an object unless the Fe abundance is severely (by a factor of $\sim 10$ ) depleted relative to solar, prompting Schmitt et al. to dub the phenomenon the "MAD" (metal abundance deficient) syndrome. However, the coronal composition might simply reflect the underlying photospheric composition as expected: Randich, Gratton \& Pallavicini (1993) have derived $\mathrm{Fe}$ abundances for the two components of $[\mathrm{Fe} / \mathrm{H}]=-0.5$ and -0.9 . Randich et al. caution that their $\mathrm{Fe}$ abundances might be systematically too low due to line filling from plage or similar emission; also, since one would expect the Fe abundances to be the same in both stars, the composition of CF Tuc warrants further investigation.

\subsection{Evidence for a FIP Effect in G-M Dwarfs-The Sources of Cosmic Rays?}

We have very recently derived abundance ratios for low and high FIP elements observed in the $E U V E$ coronal spectrum of the $\alpha$ Cen $\mathrm{AB}(\mathrm{G} 0 \mathrm{~V}+\mathrm{K} 1 \mathrm{~V})$ system using temperature insensitive line ratios (Drake, Laming \& Widing 1995b). The $\alpha$ Cen AB system was not spatially resolved by $E U V E$, and it is currently not known which component was brightest in the EUV at the time of the observation. $\dagger$ It is possible that the solar analogue and cooler $\mathrm{K}$ dwarf show different coronal abundance anomalies.

The active G8 dwarf $\xi$ Boo A also appears to exhibit a FIP effect based on an EM distribution analysis similar to that carried out for Procyon (Figure 2). Though still preliminary, this result would be extremely important because it would represent the first detection of the FIP effect in a star which is significantly cooler than the Sun. It argues strongly against a photospheric site for the FIP fractionation, since ions such as Fe II, Si II and Mg II will not be such major species in the photosphere of this star as compared to the Sun.

The same type of analysis applied to the intermediate activity K2 dwarf $\epsilon$ Eri yielded similar results, though not without some equivocation (Laming, Drake \& Widing 1995). However, Laming et al. were also able to place limits on the magnitude of the FIP effect in $\epsilon$ Eri, arguing that it could not be substantially larger than that found in the sun. This suggests that the FIP effect is not "linearly" related to stellar activity, as one might naively expect from the fact that the fractionation according to FIP must be driven by electromagnetic forces - forces directly related to stellar activity.

Preliminary results for the dMe binary FK Aqr also indicate the presence of a FIP effect. Unfortunately, the exposure was too short to detect the high FIP lines needed to quantify the result. Nevertheless, upper limits to the fluxes from these lines do indicate

$\dagger$ Golub et al. (1982) found $B$ to be brightest in X-rays from an observation in 1979. In view of the large cyclic and stochastic variability in the solar X-ray flux-by more than a factor of 10 -extrapolation of this result to the time of the EUVE observation is perilous. 


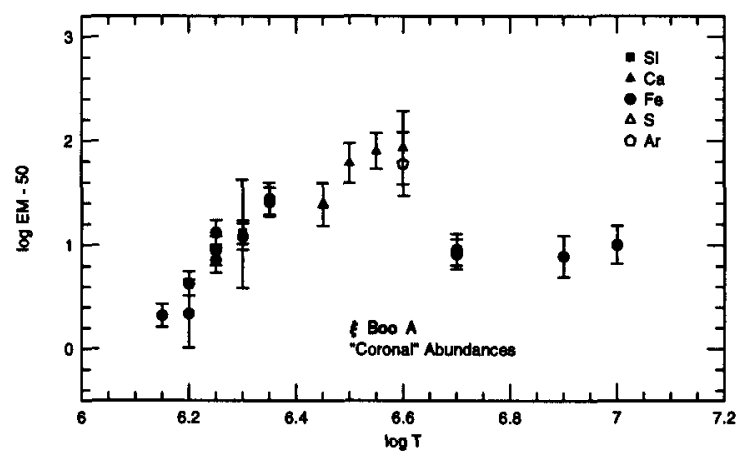

FIGURE 2. The EM distribution for low and high FIP species in the EUVE spectrum of $\xi$ Boo A for Feldman (1992) "coronal" abundances.

an enhancement of low FIP species relative to the high FIPs by at least the same amount as seen on the Sun-factors of 4 or more.

The results for these stars are of central importance for the origin of cosmic rays: we find that the types of stars proposed by Meyer (1985b) to supply the cosmic ray seed particles appear to exhibit a solar-like FIP effect. Our results therefore support the hypothesis that the galactic cosmic rays originate predominantly from the surfaces of late-type stars.

\subsection{Abundance Studies with ASCA}

In addition to the work of Antunes et al. (1994), mentioned above, Drake et al. (1994) have derived abundances using two temperature coronal models from $A S C A$ observations of $\pi^{1} \mathrm{UMa}(\mathrm{G} 1.5 \mathrm{~V}$ ) and $\beta$ Ceti (K0 III). Interestingly, they obtained a bias similar to that of a FIP-effect, but with some deviations. Most notably, Fe was observed to be depleted rather than enhanced, relative to the solar mixture. White et al. (1994) also found evidence for metal deficiency in the corona of the RS CVn system AR Lac, and pointed to similar hints from GINGA observations; they ventured that these abundances possibly just reflected the photospheric compositions of the RS CVn secondaries, which some optical observations had also suggested were metal poor. Despite the possibility that the underabundances might just reflect the stellar compositions, and also that the real uncertainties in the $A S C A$ abundances remain to be quantified, these results, and those for Algol and CF Tuc, raise some very interesting questions: is FIP not the universally common factor in coronal abundances? The depletion of Fe would be particularly mysterious and does not fit with any existing fractionation models.

\section{Summary}

Recent studies with $E U V E$ and $A S C A$ indicate that the Sun is not alone in exhibiting coronal abundance anomalies. G-M dwarfs appear to exhibit solar-like FIP effects. This is consistent with Meyer's (1985b) hypothesis that cosmic rays originate from suprathermal seed particles injected into the ISM by late-type stellar coronae.

Are the coronae of the very active stars really metal deficient ("MAD"!) relative to their photospheric compositions? If so, FIP might not be the universal underlying factor controlling coronal abundance anomalies. It is vital to know the photospheric and coronal 
compositions of the stars whose coronae we wish to study and attempt to understand. Based on the solar analogy, coronal abundance anomalies, if understood, promise exciting new diagnostics for processes occurring in stellar outer atmospheres. Future abundance studies with $A S C A$ should concentrate on stars with EM distributions determined from $E U V E$ observations.

Much longer exposures with $E U V E$ and $A S C A$ than have hitherto been the norm are essential for making the next advancements.

\section{REFERENCES}

ANDErs, E. \& Grevesse, N. 1989, Geochim. Cosmochim. Acta., 53, 197

Antunes, A., Nagase, F., \& White, N. E. 1995, ApJ, 436, L83

Axford, W. I., Leer, E., \& Skandron, K. G. 1977, Proc. 15th Int. Cosmic Ray Conf., 11, 32

Bloemen, H. 1987, in "Interstellar Processes," ed. D. J. Hollenbach \& H. A. Thronson, Jr., Reidel, 143

CAssÉ, M., \& Goret, P. 1978, ApJ, 221, 703

Cook, J. W., Cheng, C. -C., Jacobs, V. L., \& Antiochos, S. K. 1989, ApJ, 338, 1176

Drake, J. J., Lammg, J. M., \& Widing, K. G. 1995a, ApJ, 443, 393

Drake, J. J., Laming, J. M., \& Widing, K. G. 1995b, ApJ, submitted

Drake, J. J., Laming, J. M., Wming, K. G., Schmitt, J. H. M. M., Haisch, B., \& BOWYER, S. 1995, Science, 267, 1470

Drake, S. A., Singh, K. P., White, N. E., \& Simon, T. 1994, ApJ, 436, L87

Feldman, U. 1992, Phys. Scripta, 46, 202

Feldman, U. \& Laming, J. M. 1994, ApJ, 434, 370

Goldberg, L., Müller, E., \& Aller, L. H. 1960, ApJS, 5, 45

Golub, L., Harnden, F. R., Pallavicini, R., Rosner, R., \& Vaiana, G. S. 1982, ApJ, 253, 242

Gorenstein, P. 1981, in Proc. 17th Int. Cosmic Ray Conf., Paris, 12, 99

HaIsch, B., SABA, J. L. R., \& MEYER, J. -P. 1995, this proceedings

Laming, J. M., Drake, J. J., \& Widing, K. G. 1995a, ApJ, 443, 416

Laming, J. M., Drake, J. J., \& Widing, K. G. 1995b, ApJ, submitted

MEYER, J. -P. 1985a, ApJS, 57, 151

MEYER, J. -P. 1985b, ApJS, 57, 172

MEYER, J. -P. 1993, in Origin and Evolution of the Elements, ed. N. Prantzos, E. VangioniFlam, \& M. Cassé, Cambridge: Cambridge University Press

MullaN, D. J. 1979, ApJ, 234, 588

Mullan, D. J. \& Cheng, Q. Q. 1994, ApJ, 435, 435

Pottasch, S. R. 1963, ApJ, 137, 945

Randich, S., Gratton, R., \& Pallavicini, R. 1993, A\&A, 273, 194

SABA, J. L. R. 1995, Adv. Sp. Res., 15(7), 13

Schmitt, J. H. M. M., Stern, R. A., Drake, J. J., \& Kürster, M. 1995, ApJ, submitted Simon, T. \& Drake, S. A. 1989, ApJ, 346, 303

Stern, R. A., Lemen, J. R., Schmitt, J. H. M. M., \& Pye, J. P. 1995, ApJ, 444, L45

TolstoY, L. N. 1889, The Kreutzer Sonata

von Steiger, R., \& Geiss, J. 1989, A\&A, 225, 222

White, N. E., ET AL. 1994, PASJ, 46, L97 